\title{
Synthesis of Atenolol-Imprinted Polymers with Methyl Methacrylate as Functional Monomer in Propanol Using Bulk and Precipitation Polymerization Method
}

\author{
Aliya Nur Hasanah (D), Traju Ningtias Dwi Utari, and Rimadani Pratiwi \\ Department of Pharmaceutical Analysis and Medicinal Chemistry, Faculty of Pharmacy, Padjadjaran University, \\ Jl. Raya Bandung Sumedang KM 21,5 Jatinangor, Sumedang, Indonesia
}

Correspondence should be addressed to Aliya Nur Hasanah; aliya.n.hasanah@unpad.ac.id

Received 11 February 2019; Accepted 24 March 2019; Published 19 May 2019

Academic Editor: Núria Fontanals

Copyright ( 2019 Aliya Nur Hasanah et al. This is an open access article distributed under the Creative Commons Attribution License, which permits unrestricted use, distribution, and reproduction in any medium, provided the original work is properly cited.

\begin{abstract}
Atenolol is one of the beta- 1 blocker drugs that is misused by athletes to increase their performance during competition. Therefore, it is important to analyze atenolol levels in blood selectively. The preparation method that can be used in separating atenolol in sample is molecular imprinting solid-phase extraction (MI-SPE) because it has good selectivity and sensitivity. This study aims to examine the characteristics and analytical performance of imprinted polymers synthesized from functional monomer methyl methacrylate. The stages of this study include the determination of association constants, synthesis of sorbent MI-SPE atenolol using the bulk polymerization method, and precipitation with atenolol as the template, methyl methacrylate as the functional monomer, and propanol as the porogen. The template was extracted from a polymer, and then, the adsorption ability, capacity, and selectivity of MI-SPE and finally the application of the best MI-SPE to spiked serum samples were determined. MI-SPE was also characterized by using Fourier-transform instrument infrared (FTIR) and scanning electron microscope (SEM). The result of characterization with FTIR and SEM showed that MIP made by the precipitation polymerization method was completely polymerized, more porous, and produced smaller particle size with an average value of $0.274 \mu \mathrm{m}$. It had better analytic performances than MIP made by bulk polymerization, with affinity value $0.3607 \mathrm{mg} / \mathrm{g}$ and homogeneity value 1.3246, and good selectivity toward atenolol with imprinting factor value 22.519. Application of MI-SPE to spiked serum samples has an excellent recovery percentage of $95.46 \%$ over $0 \%$ for the nonimprinting one. Based on the result of study, MIP made by precipitation polymerization could be used to extract atenolol on serum samples toward drug analysis.
\end{abstract}

\section{Introduction}

Atenolol is an adrenoreceptor inhibitor that is selective to $\beta-1$, and it is used to treat hypertension, cardiac arrhythmias, angina pectoris, myocardial infarction, and other cardiovascular disorders [1]. Atenolol is often misused by athletes to improve their performance because it reduces heart rate and tremors. Thus, the use of atenolol is prohibited by the World AntiDoping Agency (WADA) and categorized as doping drugs. Therefore, monitoring levels of atenolol in blood is very important. A selective extraction method is needed because atenolol is in a complicated sample matrix [2]. The preparation method that is currently developing and has good selectivity is molecularly imprinted solid-phase extraction (MI-SPE). Molecular-imprinting is a technique for preparing polymeric materials that have prearranged structures and specific molecular recognition ability [3].

In this case, the selection of functional monomer and porogen has important role in producing molecular-specific cavities of templates. Methyl methacrylate is a functional monomer that can act as a hydrogen bond acceptor to its template [4]. Other researchers have done research on MISPE for atenolol [5-7], but none of them used precipitation polymerization as we used in this study, and the recoveries are below $80 \%$. The porogen chosen in this study is propanol because it has low polarity that can reduce interference 
during the polymerization process and increase the number of MIP-binding sites [8].

In this study, we describe the synthesis of MIP for extraction of atenolol and for use as SPE sorbents for the selective extraction of atenolol in blood samples using two different methods. The methods are precipitation and bulk polymerization. The polymer was synthesized and characterized for the ability of the polymer to bind with atenolol selectively. The MIP was synthesized using methyl methacrylate as a functional monomer, ethylene glycol dimethacrylate as a cross linker, propanol as a porogenic solvent, and benzoyl peroxide as an initiator. The binding affinity of the binding sites in the polymer was assessed using the adsorption test. The characterization was carried out using a Fourier-transform infrared (FTIR) spectroscope and scanning electron microscope (SEM). At the end, this polymer was then used as an extraction material of atenolol from the spiked blood serum.

\section{Materials and Methods}

2.1. Materials. Acetonitrile was purchased from Fisher Chemical; atenolol, metoprolol, and propranolol were purchased from TCI. Acetic acid 96\%, benzoyl peroxide (BPO), methanol, potassium bromide, and propanol were purchased from Merck. Ethylene glycol dimethacrylate (EGDMA) and methyl methacrylate (MMA) were purchased from Sigma-Aldrich. All chemicals used were commercially available and were of analytical grade. Methanol used was of HPLC gradient grade. Methyl methacrylate was purified before being used with aluminium chloride.

\subsection{Determination of Association Constant of Monomer} Template. Atenolol $2 \times 10^{-5} \mathrm{M}$ was diluted in propanol. $2.5 \mathrm{ml}$ of the solution was measured using a UV-Vis spectrophotometer. Methyl methacrylate solution of $0.01 \mathrm{M}$ was added gradually from $10 \mu \mathrm{l}, 20 \mu \mathrm{l}, 30 \mu \mathrm{l}, 40 \mu \mathrm{l}, 50 \mu \mathrm{l}, 100 \mu \mathrm{l}$, and so on, until there was no significant increase in the absorbance value. The results are plotted on a graph between $1 /[G]$ and $1 / \Delta Y$. The constant association was calculated using Benesi-Hildebrand equation [9].

$$
\frac{1}{\Delta Y}=\frac{1}{Y \Delta \mathrm{HGKa}[G]}+\frac{1}{Y \Delta \mathrm{HG}},
$$

where $\Delta Y$ is the change in absorbance, $Y \Delta H G$ is the change in absorbance at the end of titration, and $[G]$ is the concentration added [10].

\subsection{Synthesis of Atenolol-Imprinted Polymer Using Bulk} Polymerization. A prepolymerization solution consisting of $0.263 \mathrm{~g}$ atenolol, $400 \mu \mathrm{l} \mathrm{MMA}, 3.77 \mathrm{ml}$ EGDMA, $250 \mathrm{mg}$ $\mathrm{BPO}$, and $5 \mathrm{ml}$ propanol was prepared in a vial. The reaction molar ratio of the template, functional monomer, and cross linker for the preparation of MIP was $1: 4: 20$. The solution was sonicated for 20 minutes to remove oxygen. The atenolol-MIP was placed in an oven at $70^{\circ} \mathrm{C}$ for 1 hour and moved to water bath and kept at $70^{\circ} \mathrm{C}$ for 18 hours for polymerization. The resulting bulk polymers were ground and sieved using mesh 60, washed using $20 \mathrm{ml}$ methanol, and dried at $50^{\circ} \mathrm{C}$. The nonimprinted polymer (NIP) was prepared simultaneously under the same condition without the addition of the template [8-11].

2.4. Synthesis of Atenolol-Imprinted Polymer Using Precipitation Polymerization. A prepolymerization solution consisting of $0.133 \mathrm{~g}$ atenolol, $200 \mu \mathrm{l}$ MMA, $1.885 \mathrm{ml}$ EGDMA, $750 \mathrm{mg}$ BPO, and $175 \mathrm{ml}$ propanol was prepared in a glass vial. The reaction molar ratio of the template, functional monomer, and cross linker for the preparation of MIP was $1: 4: 20$. The solution was sonicated for 20 minutes to remove oxygen. The atenolol-MIP was placed in an oven at $70^{\circ} \mathrm{C}$ for 1 hour and moved to water bath shaker and kept at $70^{\circ} \mathrm{C}$ for 18 hours for polymerization. The resulting bulk polymers were washed using $40 \mathrm{ml}$ methanol and dried at $50^{\circ} \mathrm{C}$. The nonimprinted polymer (NIP) was prepared simultaneously under the same condition without the addition of template [9].

2.5. Extraction of Template. The Soxhlet apparatus was used for template removal from the synthesized MIP using methanol and acetic acid $(9: 1)$ for 24 hours. Then, the polymers were washed using $20 \mathrm{ml}$ methanol and water and dried at $50^{\circ} \mathrm{C}$ for 18 hours.

MIP was monitored using $20 \mathrm{mg}$ MIP diluted in $5 \mathrm{ml}$ methanol, triplicate. Then, MIP was sonicated for 5 minutes and set aside for 24 hours. The extraction process is complete when the liquid leaching results in MIP no longer contain the template when monitored using a UV-Vis spectrophotometer [11].

2.6. Evaluation of Binding Ability. Twenty milligrams of MIP in vial was incorporated into $5 \mathrm{ml}$ atenolol at a concentration of $5 \mathrm{ppm}$ in various solutions such as methanol, acetonitrile, and methanol:acetonitrile $(1: 1)$, triplicate. The vials were shaken using a shaker at $120 \mathrm{rpm}$ for 3 hours at room temperature to reach equilibrium and then filtered. The filtrate was measured by using a UV-Vis spectrophotometer. The evaluation of NIP was carried out by the same procedure as that for MIP [11].

2.7. Evaluation of Binding Capacity. Five milliliters of selected solvent from evaluation 2.6 containing $2.5 ; 5 ; 7.5$; $10 \mathrm{ppm}$ of atenolol was added to $20 \mathrm{mg}$ of MIP in vials, triplicate. The vials were shaken using a shaker at $120 \mathrm{rpm}$ for 3 hours at room temperature and then filtered. The filtrate was measured by using a UV-Vis spectrophotometer. The results are plotted for isotherm Freundlich adsorption curve. The adsorption curve of NIP was plotted by the same procedure as that for MIP $[11,12]$.

2.8. Determination of Selectivity. Atenolol, metoprolol, and propranolol were used to determine the relative selectivity of MIP. $5 \mathrm{ml}$ of selected solvent from evaluation 2.6 containing 
$5 \mathrm{ppm}$ of atenolol; metoprolol; propranolol was added to $20 \mathrm{mg}$ of MIP in vials, triplicate. The vials were shaken using a shaker at $120 \mathrm{rpm}$ for 3 hours at room temperature and then filtered. The filtrate was measured by using a UV-Vis spectrophotometer. The evaluation of NIP was carried out by the same procedure as that for MIP. The imprinting factor was calculated using the following equation:

$$
\begin{gathered}
K_{\mathrm{D}}=\frac{\left(C_{\mathrm{i}}-C_{\mathrm{f}}\right) V}{C_{\mathrm{f}} m}, \\
\mathrm{IF}=\frac{K_{\mathrm{D}} \mathrm{MIP}}{K_{\mathrm{D}} \mathrm{NIP}},
\end{gathered}
$$

where $K_{\mathrm{D}}$ is the distribution coefficient; $C_{\mathrm{i}}$ and $C_{\mathrm{f}}$ are the concentration of atenolol before and after the adsorption experiments, respectively; $V$ is the volume of solution containing atenolol; and $W$ is the weight of the polymer $[13,14]$.

2.9. Application of the Polymer in Serum Samples. The blood serum is obtained by centrifugation of blood at a speed of $5000 \mathrm{rpm}$ for 5 minutes; then the supernatant is collected. The blood serum is spiked with $2 \mathrm{ppm}$ atenolol in water. The spiked serum is passed into MIP-SPE and NIP-SPE. The SPE system is conditioned with methanol:acetonitrile $(1: 1)$ $3 \times 1 \mathrm{~mL}$, washing solvents using acetonitrile, and elution using methanol: trifluoroacetic acid $0.05 \%(99: 1) 3 \times 1 \mathrm{~mL}$. The elution results were then analyzed by HPLC using the mobile phase of methanol: water + triethylamine $0.05 \%$ which was adjusted to $\mathrm{pH} 3$ with phosphoric acid $(15: 85)$.

2.10. Characterization of Atenolol-Imprinted Polymer. The chemical structure of MIP and NIP samples was characterized by FTIR spectroscopy (IRPrestige-21, Shimadzu). Samples were ground and pressed into $\mathrm{KBr}$ plates. The analysis was performed between 400 and $4000 \mathrm{~cm}^{-1}$. The surface morphology was analyzed by SEM $[11,15,16]$.

\section{Results and Discussion}

3.1. Determination of Association Constant of Monomer Template. Before the polymerization step, the association constant was determined to know the ability of MMA functional monomer to bind with atenolol to form a stable complex in prepolymerization solution with the titration method using a UV-Vis spectrophotometer [17].

The association constant was $199.625 \mathrm{M}^{-1}$, calculated by Benesi-Hildebrand equation (Figure 1). The higher the value of the association constant, the more stable the complex that occurs during polymerization and the better the imprinting effect $[18,19]$.

\subsection{Synthesis of Atenolol-Imprinted Polymer Using Bulk and} Precipitation Polymerization. The purpose of the synthesis by two methods is to see the effectiveness of each polymer produced. In molecular-imprinting processes, the selection of the functional monomer is an important factor that affects

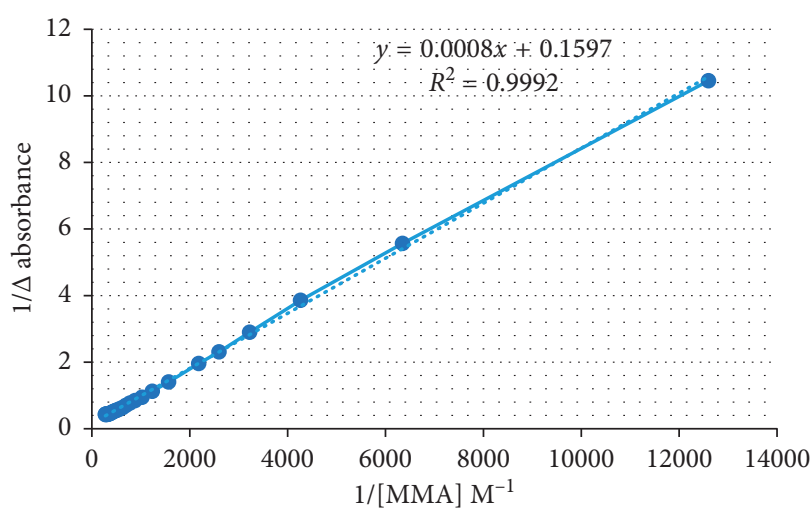

FIgURE 1: Relationship between $1 /$ (methyl methacrylate) to $1 /$ $\Delta$ absorbance.

the binding affinity and specificity of the imprinted polymer. The formulations were prepared by the bulk and precipitation polymerization method using MMA as the monomer, BPO as the initiator, and EGDMA as the cross linker. The ratio of the monomer affected the particle sizes and \% yields of the obtained MIP and NIP [20].

3.3. Extraction of Template. The purpose of extraction was to remove atenolol groups that bind to polymers and to form cavities that were complementary to atenolol [18]. Atenolol is soluble in methanol, so it was used to extract the template. Acetic acid was added to disrupt the hydrogen bond between atenolol and the functional monomer MMA to facilitate the removal of atenolol $[12,21]$.

3.4. Evaluation of Binding Ability. In order to know the binding ability and to find out the optimum conditions for the template to be recognized by the MIP that is being prepared, a standard solution of atenolol of $5 \mathrm{ppm}$ was initially prepared in various solvents such as methanol, acetonitrile, and methanol:acetonitrile $(1: 1)$. The filtrate that indicated the amount of unbound analyte was measured. The atenolol-binding ability of MIPs was investigated and compared with that of NIPs [15].

From Figure 2, it is known that the MIP synthesized using the bulk polymerization method can bind with atenolol in acetonitrile, with $31.854 \%$ of binding. However, NIPs in other solvents such as methanol and methanol:acetonitrile $(1: 1)$ showed a higher percent of binding, $89.908 \%$ and $39.483 \%$, respectively. This suggests that NIPs swelled better in these solvents. From Figure 3, the MIP synthesized using the precipitation polymerization method can bind atenolol in methanol: acetonitrile $(1: 1)$, with $38.543 \%$ of binding. This showed that acetonitrile has the ability to lead atenolol properly to the binding site in MIP $[13,22,23]$.

3.5. Evaluation of Binding Capacity. This evaluation is used to study the affinity between MIP and analyte target [23]. The relationship between the amount of analyte bound to the adsorbent $(B)$ and the amount of free analyte $(F)$ is illustrated in the following Freundlich isotherm equation: 


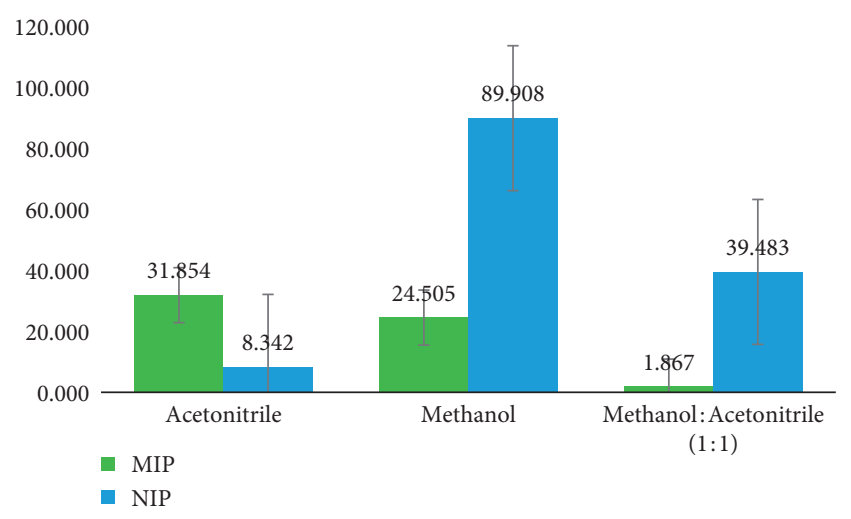

Figure 2: Binding ability MMA propanol polymer bulk method $(n=3)$.

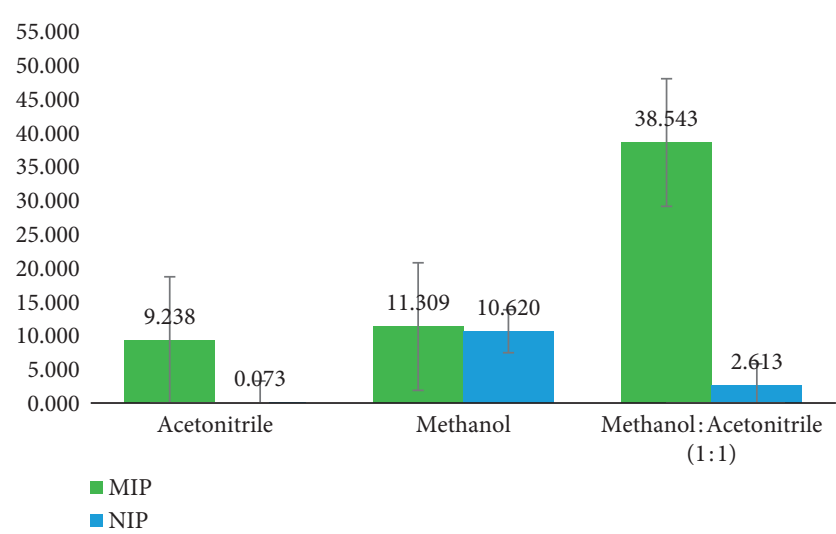

FIgURE 3: Binding ability MMA propanol polymer precipitation method $(n=3)$.

$$
\log B=m \log F+\log a .
$$

The value of $m$ indicates the homogeneity index; when it approaches 1 , the sorbent is more homogeneous, and when it approaches 0 , the sorbent is more heterogeneous [13]. The value of $a$ indicates sorbent affinity; the higher the affinity obtained, the more the capacity of the sorbent to bind to the analyte target $[24,25]$. Table 1 shows the binding capacity value of each MIP and NIP. The MIP that was synthesized using the precipitation method has a homogeneity value close to 1 , which is 1.3426 , and an affinity value of 0.3607 . It has better $a$ and $m$ values than the MIP that was synthesized by bulk polymerization, which indicates better imprinting effect.

3.6. Determination of Selectivity. The selectivity for atenolol of MIP was investigated by determining its binding ability compared with other $\beta$-blocker drugs such as metoprolol and propranolol. A testing solution containing $5 \mathrm{ppm}$ of each drug (atenolol, metoprolol, and propranolol) was prepared [17]. MIP and NIP were prepared $20 \mathrm{mg}$ in each vial. Solution and MIP were combined in a vial then shaken using a shaker to reach equilibrium. The filtrate was measured using a UV-Vis spectrophotometer. The distribution coefficient $\left(C_{\mathrm{D}}\right)$ and imprinting factor (IF) were calculated. The results (Tables 2 and 3 ) showed that atenolol can bind
TABLE 1: Binding capacity MMA propanol bulk and precipitation method $(n=3)$.

\begin{tabular}{lcccc}
\hline Value & \multicolumn{2}{c}{ MMA propanol bulk } & \multicolumn{2}{c}{$\begin{array}{c}\text { MMA propanol } \\
\text { precipitation }\end{array}$} \\
& MIP & NIP & MIP & NIP \\
\hline$M$ & -0.1909 & 0.102 & 1.3246 & 2.3672 \\
$a(\mathrm{mg} / \mathrm{g})$ & 0.07 & 0.0872 & 0.3607 & 0.0028 \\
$R^{2}$ & 0.0198 & 0.0182 & 0.9547 & 0.8061 \\
\hline
\end{tabular}

TABLE 2: Selectivity of MMA propanol polymer by bulk polymerization $(n=3)$.

\begin{tabular}{|c|c|c|c|c|}
\hline \multicolumn{2}{|c|}{ Analyte } & Atenolol & Propranolol & Metoprolol \\
\hline \multirow{2}{*}{$K_{\mathrm{D}}(\mathrm{ml} / \mathrm{g})$} & MIP & 115.385 & 218.624 & 0.170 \\
\hline & NIP & 22.135 & 332.559 & 0.022 \\
\hline \multicolumn{2}{|c|}{ Imprinting factor } & 5.213 & 0.657 & 7.869 \\
\hline
\end{tabular}

TABLE 3: Selectivity of MMA propanol polymer by precipitation polymerization $(n=3)$.

\begin{tabular}{|c|c|c|c|c|}
\hline \multicolumn{2}{|c|}{ Analyte } & Atenolol & Propranolol & Metoprolol \\
\hline \multirow{2}{*}{$K_{\mathrm{D}}(\mathrm{ml} / \mathrm{g})$} & MIP & 156.863 & 97.32 & 334.172 \\
\hline & NIP & 6.966 & 176.443 & 144.475 \\
\hline \multicolumn{2}{|c|}{ Imprinting factor } & 22.519 & 0.553 & 2.313 \\
\hline
\end{tabular}

with MIP synthesized by the precipitation method with a distribution coefficient of $156.864 \mathrm{ml} / \mathrm{g}$, which is higher than that of NIP which was $6.966 \mathrm{ml} / \mathrm{g}$. The imprinting factor of MIP was 22.519. This indicates that the MIP that was synthesized by the precipitation method had a higher selectivity for atenolol than the other $\beta$-blocker drugs. Thus, it was selective for atenolol.

3.7. Application of the Polymer in Serum Samples. The polymer made by precipitation polymerization has better analytical performances compared to bulk. Based on this result, a $100 \mathrm{mg}$ polymer made by precipitation polymerization then was packed into cartridges and used as an SPE material over the spiked blood serum. The imprinting polymer gain recovery percentage was $95.46 \% \pm 3.44 \%$ compared to nonimprinted $0.00 \% \pm 0.00 \%$. This result showed an excellent imprinting effect and was linear to the selectivity result which had an imprinting factor value of 22.519 compared with NIP. All experiments were done using the same cartridges (over 20 cycles), and the results are still in the acceptance range (SD below 5\%). The result of this polymer is better than that of the one in the previous study [7] and fits with criteria recoveries of analyte from biological fluid Table 4.

3.8. Characterization of Atenolol-Imprinted Polymer. The FTIR spectrums of the MIP by bulk and precipitation polymerization are presented in Figures 4 and 5. The complete polymerization process was characterized when the doublet peak (vinyl group, $\mathrm{H}_{2} \mathrm{C}=\mathrm{CH}-$ ) was absent at the 
TABLE 4: Result of application of the polymer in serum samples compared to previous study [7].

\begin{tabular}{lcr}
\hline & Polymer MMA made by precipitation polymerization & Polymer from previous study [7] \\
\hline Imprinting factor (IF) & 22.519 & 4.18 \\
Recoveries & $95.46 \%$ & $74.5-75.3 \%$ \\
\hline
\end{tabular}

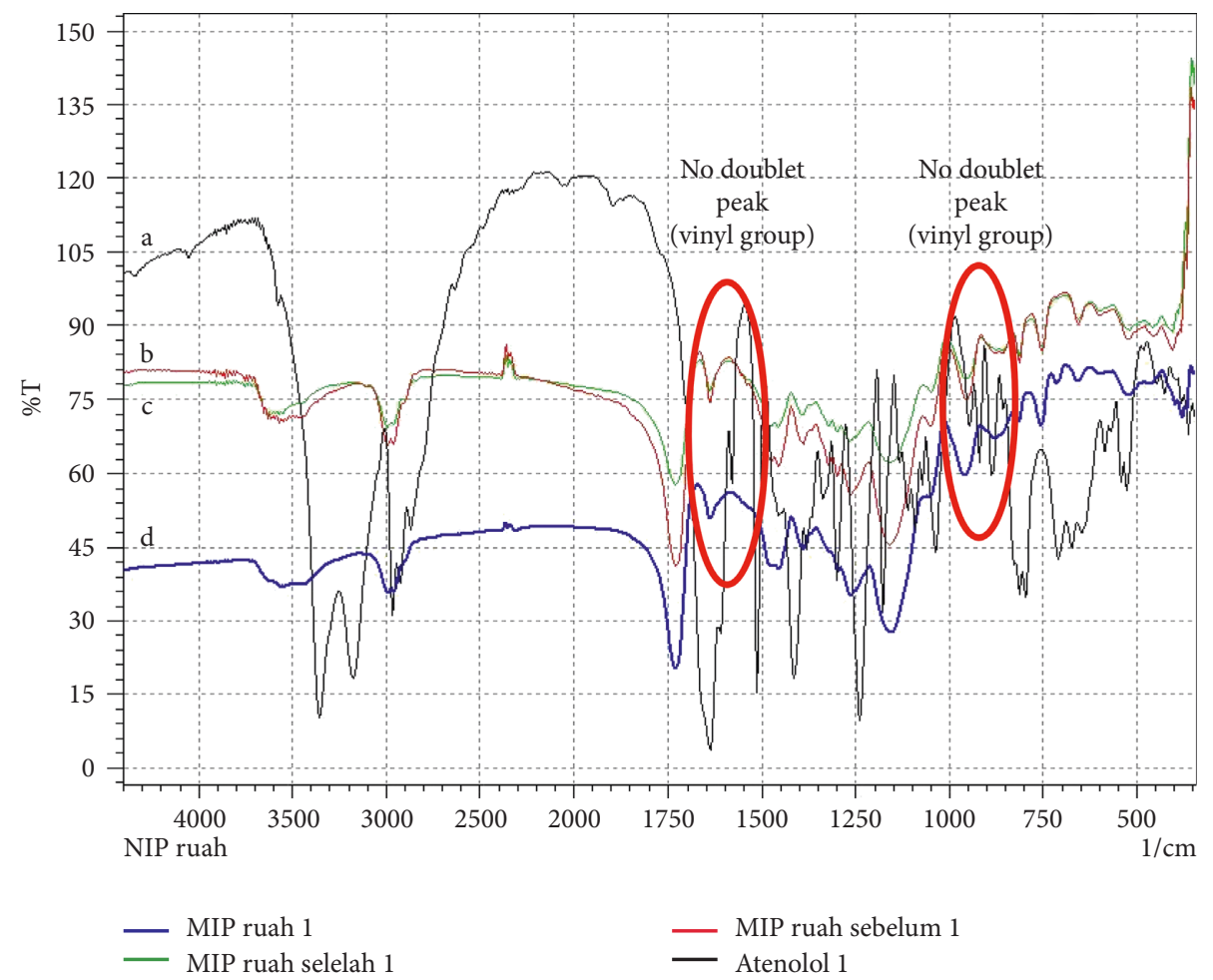

FIGURE 4: FTIR spectrum: (a) atenolol; (b) MIP by bulk before extraction; (c) MIP by bulk after extraction; (d) NIP by bulk.

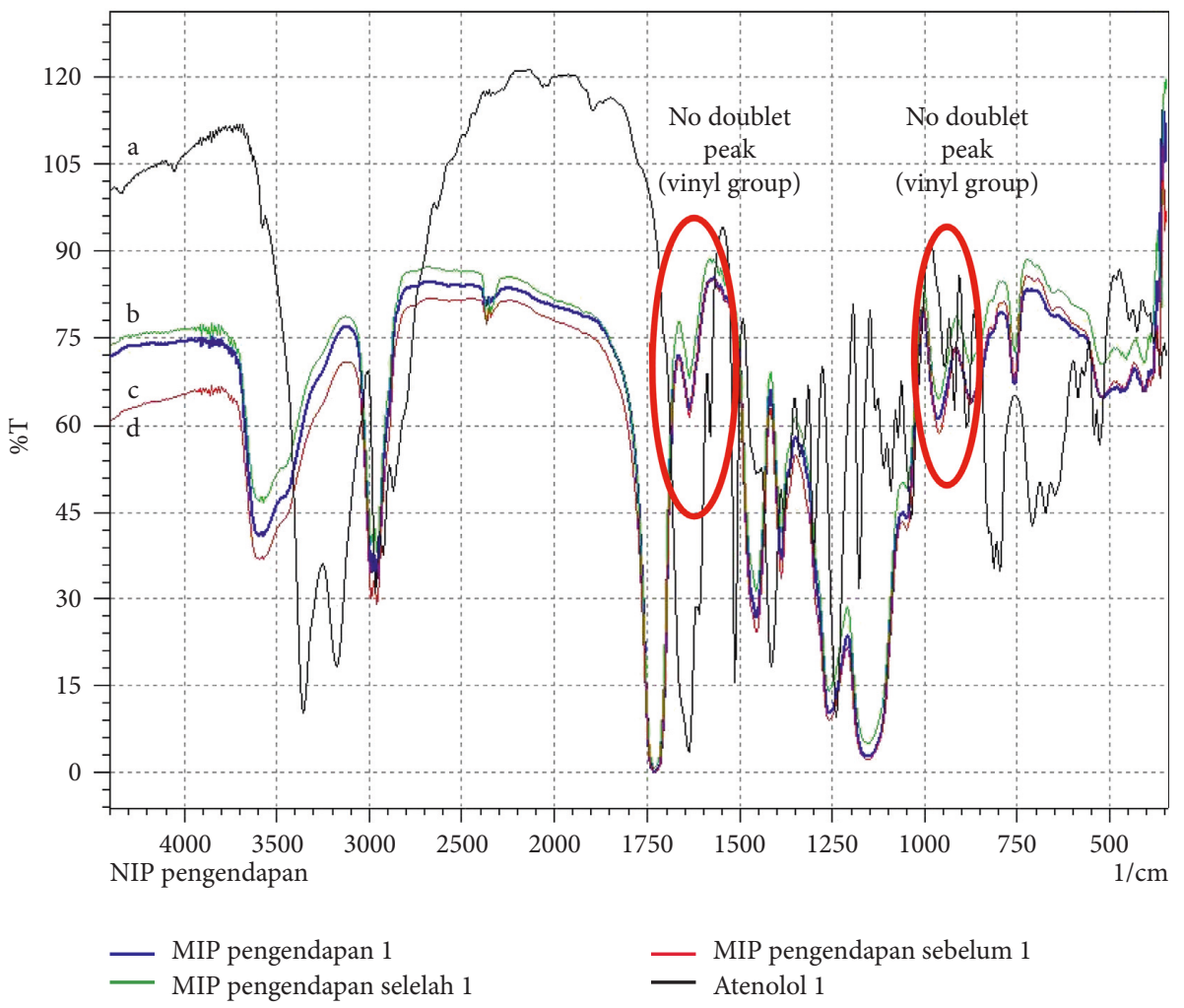

FIGURE 5: FTIR spectrum: (a) atenolol; (b) MIP by precipitation after extraction; (c) NIP by precipitation; (d) MIP by precipitation before extraction. 


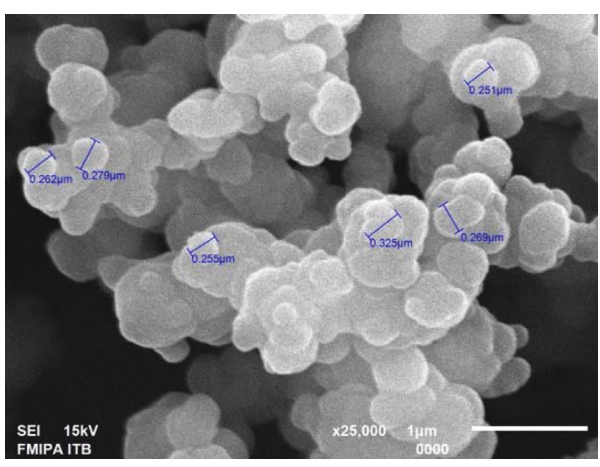

(a)

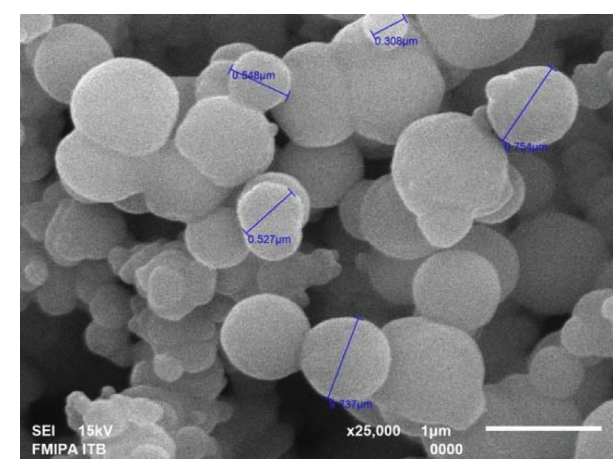

(b)

FIgURE 6: SEM images of (a) MIP by precipitation polymerization and (b) NIP by precipitation polymerization.

wavenumber $1600 \mathrm{~cm}^{-1}, 1660-1635 \mathrm{~cm}^{-1}, 990 \pm 5 \mathrm{~cm}^{-1}$, and $910 \pm 5 \mathrm{~cm}^{-1}[26]$.

The morphologies and particle size of MIP and NIP that were synthesized by precipitation polymerization were determined using SEM and the results are shown in Figure 6. The SEM images of the NIP revealed a spherical and smooth surface without evidence of collapsed particles, whereas those of the MIP revealed spheres with small pores on the surface [17]. MIP had smaller particles; it suggested that the template compound has an important influence on the particle growth during the precipitation polymerization [20].

\section{Conclusions}

The molecularly imprinted polymer of atenolol with MMA as the functional monomer in propanol using the precipitation polymerization synthesis method had better analytic performances than MIP that was synthesized using the bulk polymerization method. The value of affinity was $0.3607 \mathrm{mg} / \mathrm{g}$, and the value of homogeneity was 1.3246 , selective toward atenolol with an imprinting factor of 22.519. Application of MI-SPE to spiked serum samples has an excellent recovery percentage of $95.46 \%$ over $0 \%$ for the nonimprinting one. The results of characterization also showed that the MIP that was synthesized using the precipitation method had small-sized homogenic particles.

\section{Data Availability}

The data used to support the findings of this study are included within the article.

\section{Conflicts of Interest}

The authors declare that there are no conflicts of interest regarding the publication of this paper.

\section{Acknowledgments}

Funding from the Ministry of Research, Technology and Higher Education Republic of Indonesia through Penelitian Terapan Unggulan Perguruan Tinggi (PTUPT) 2018, through Grant no. 381c/UN6.O/LT/2018 is greatly acknowledged.

\section{References}

[1] P. Gupta and R. N. Goyal, "Amino functionalized graphene oxide and polymer nanocomposite based electrochemical platform for sensitive assay of anti-doping drug atenolol in biological fluids," Journal of the Electrochemical Society, vol. 163, no. 13, pp. B601-B608, 2016.

[2] A. Beltran, F. Borrull, R. M. Marcé, and P. A. G. Cormack, "Molecularly imprinted polymers: useful sorbents for selective extractions," TrAC-Trends in Analytical Chemistry, vol. 29, no. 11, pp. 1363-1375, 2010.

[3] H. A. Panahi, E. Moniri, M. Aliasghari, and L. Hajaghababaii, "Selective sorption and determination of atenolol in pharmaceutical and biological samples by molecular imprinting using new copolymer beads as functional matric," Journal of Liquid Chromatography \& Related Technologies, vol. 38, no. 2, pp. 222-228, 2014.

[4] K. Golker, B. Karlsson, A. Rosengren, and I. Nicholls, “A functional monomer is not enough: principal component analysis of the influence of template complexation in prepolymerization mixtures on imprinted polymer recognition and morphology," International Journal of Molecular Sciences, vol. 15, no. 11, pp. 20572-20584, 2014.

[5] A. E. Bodoki, B.-C. Iacob, L. E. Gliga et al., "Improved enantioselectivity for atenolol employing pivot based molecular imprinting," Molecules, vol. 23, no. 8, p. 1875, 2018.

[6] T. Alizadeh, "Synthesis of a nano-sized chiral imprinted polymer and its use as an (S)-atenolol carrier in the bulk liquid membrane," Journal of Separation Science, vol. 37, no. 14, pp. 1887-1895, 2014.

[7] Y. Gorbani, H. Yılmaz, and H. Basan, "Spectrofluorimetric determination of atenolol from human urine using highaffinity molecularly imprinted solid-phase extraction sorbent," Luminescence, vol. 32, no. 8, pp. 1391-1397, 2017.

[8] H. Yan and K. Row, "Characteristic and synthetic approach of molecularly imprinted polymer," International Journal of Molecular Sciences, vol. 7, no. 5, pp. 155-178, 2006.

[9] S. Song, A. Wu, X. Shi, R. Li, Z. Lin, and D. Zhang, "Development and application of molecularly imprinted polymers as solid-phase sorbents for erythromycin extraction," Analytical and Bioanalytical Chemistry, vol. 390, no. 8, pp. 2141-2150, 2008.

[10] P. Thordarson, "Determining association constants from titration experiments in supramolecular chemistry," Chemical Society Reviews, vol. 40, no. 3, pp. 1305-1323, 2011.

[11] A. N. Hasanah, R. E. Kartasasmi, and S. Ibrahim, "Synthesis and application of glibenclamide imprinted polymer for solid 
phase extraction in serum samples using itaconic acid as functional monomer," Journal of Applied Sciences, vol. 15, no. 11, pp. 1288-1296, 2015.

[12] S. Ansari and A. Ghorbani, "Molecularly imprinted polymers (MIP) for selective solid phase extraction of celecoxib in urine sampeles followed by high performance liquid chromatography," Journal of Chemical Health Risks, vol. 7, 2017.

[13] R. J. Ansell, "Characterization of the binding properties of molecularly imprinted polymers," Advances in Biochemical Engineering Biotechnology, vol. 10, no. 7, 2015.

[14] P. Liu, L. Liu, L. Zhang, N. Jiang, Z. Liu, and Y. Wang, "Synthesis and characterization of molecularly imprinted polymers for recognition of ciprofloxacin," Frontiers of Chemistry in China, vol. 3, no. 4, pp. 378-383, 2008.

[15] A. N. Hasanah et al., "Study of the binding ability of molecular imprinted solid phase extraction for glibenclamide by optimizing template: monomer: crosslinker ratio," International Journal of Chemical Science, vol. 12, no. 3, pp. 1288-1296, 2014.

[16] J. N. T. Nguyen and A. M. Harbison, "Scanning Electron microscopy sample preparation and imaging," Methods in Molecular Biology, vol. 1606, pp. 71-84, 2017.

[17] S. Scorrano, L. Mergola, R. Del Sole, and G. Vasapollo, "Synthesis of molecularly imprinted polymers for amino acid derivates by using different functional monomers," International Journal of Molecular Sciences, vol. 12, no. 3, pp. 1735-1743, 2011.

[18] S. N. N. S. Hashim, R. I. Boysen, L. J. Schwarz, B. Danylec, and M. T. W. Hearn, "A comparison of covalent and non-covalent imprinting strategies for the synthesis of stigmasterol imprinted polymers," Journal of Chromatography A, vol. 1359, pp. 35-43, 2014.

[19] K. Lim and C. Holdsworth, "Effect of formulation on the binding efficiency and selectivity of precipitation molecularly imprinted polymers," Molecules, vol. 23, no. 11, p. 2996, 2018.

[20] P. Tonglairoum, W. Chaijaroenluk, T. Rojanarata, T. Ngawhirunpat, P. Akkaramongkolporn, and P. Opanasopit, "Development and characterization of propranolol selective molecular imprinted polymer composite electrospun nanofiber membrane," AAPS PharmSciTech, vol. 14, no. 2, pp. 838-846, 2013.

[21] L. Chen, "Molecular imprinting: perspectives and applications," Royal Society of Chemistry Advances Review Article, vol. 45 , no. 8 , pp. $1-75,2016$.

[22] F. Omidi, M. Behbahani, S. Samadi, A. Sedighi, and S. J. Shahtaheri, "Coupling of molecular imprinted polymer nanoparticles by high performance liquid chromatography as an efficient technique for sensitive and selective trace determination of 4-chloro-2-methylphenoxy acetic acid in complex matrices," Iranian Journal of Public Health, vol. 43, no. 5, pp. 645-657, 2014.

[23] C. Alvarez-Lorenzo and A. Concheiro, Handbook of Molecularly Imprinted Polymers, Smithers Rapra Technology Ltd., Shawbury, UK, 2013.

[24] G. T. Rushton, C. L. Karns, and K. D. Shimizu, "A critical examination of the use of the Freundlich isotherm in characterizing molecularly imprinted polymers (MIPs)," Analytica Chimica Acta, vol. 528, no. 1, pp. 107-113, 2005.

[25] W. Cai and R. B. Gupta, "Molecularly-imprinted polymers selective for tetracycline binding," Separation and Purification Technology, vol. 35, no. 3, pp. 215-221, 2004.

[26] B. C. Smith, Infrared Spectral Interpretation: A Systematic Approach, CRC Press LLC, Boca Raton, FL, USA, 1999. 

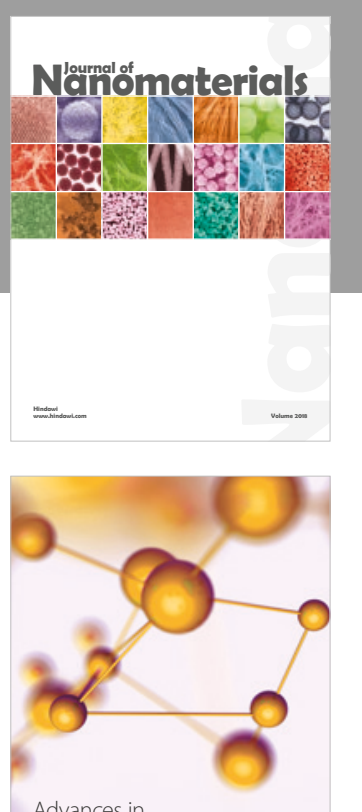

Physical Chemistry
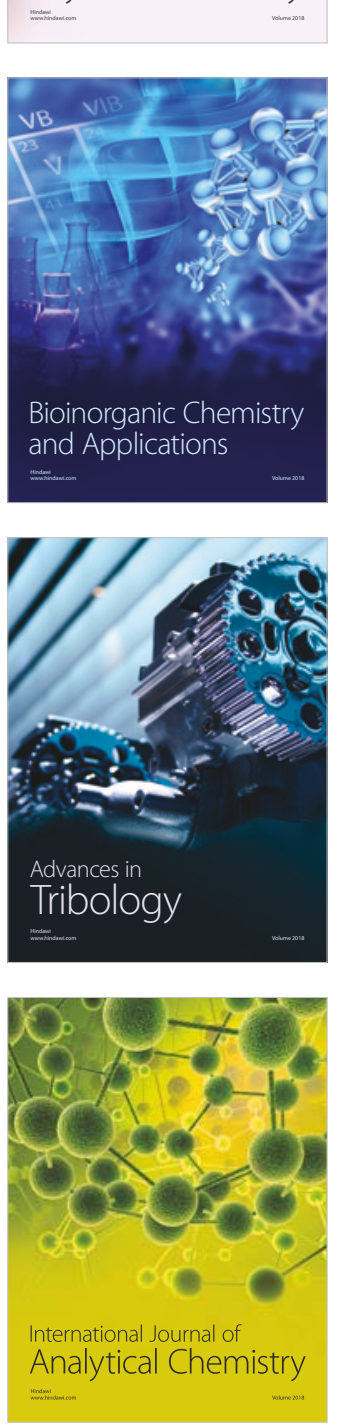

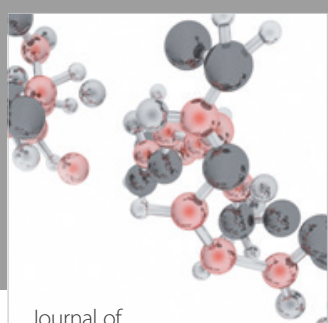

Analytical Methods

in Chemistry

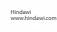

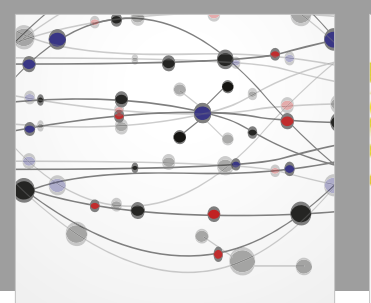

The Scientific World Journal

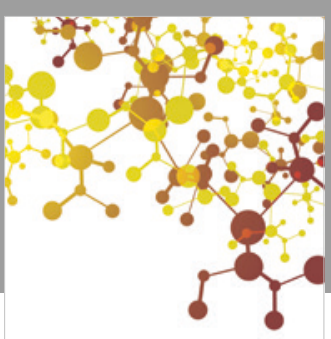

Journal of

Applied Chemistry
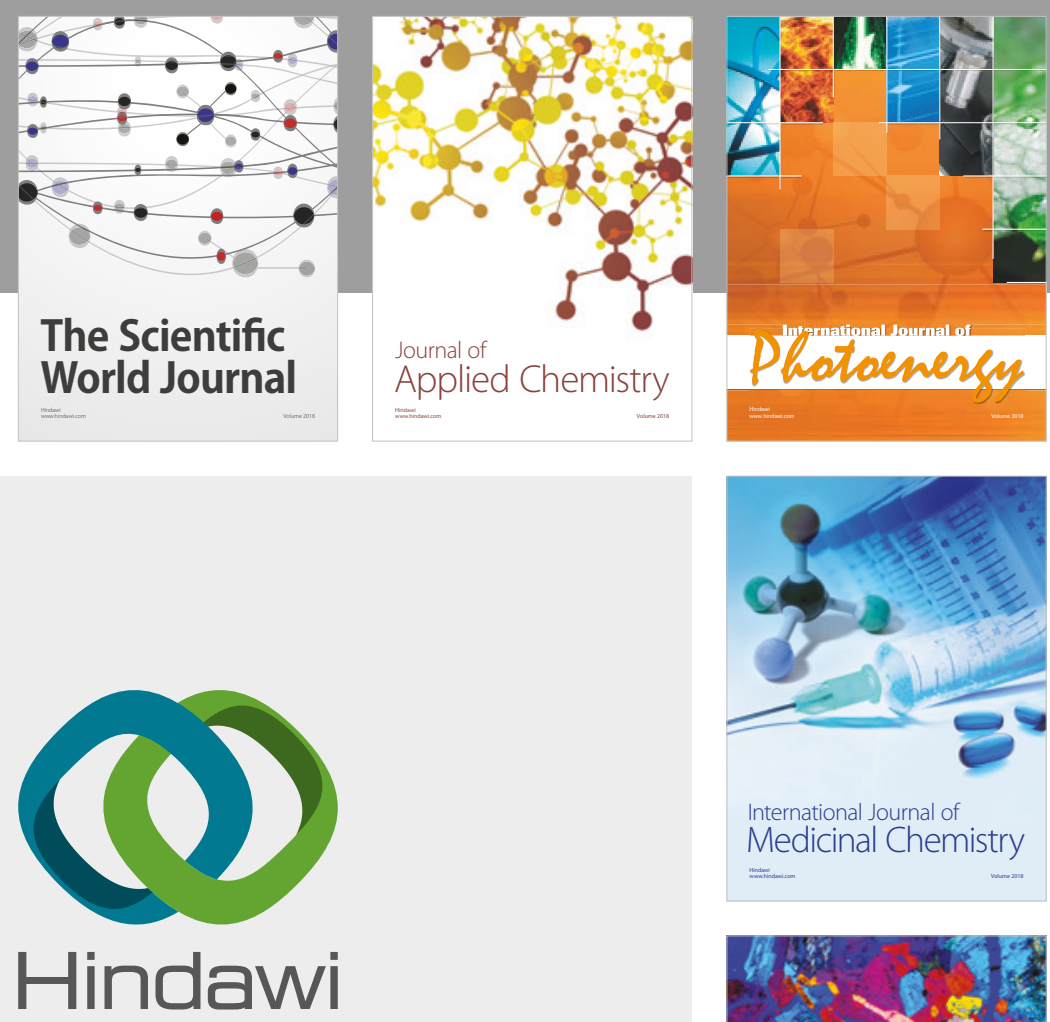

Submit your manuscripts at

www.hindawi.com
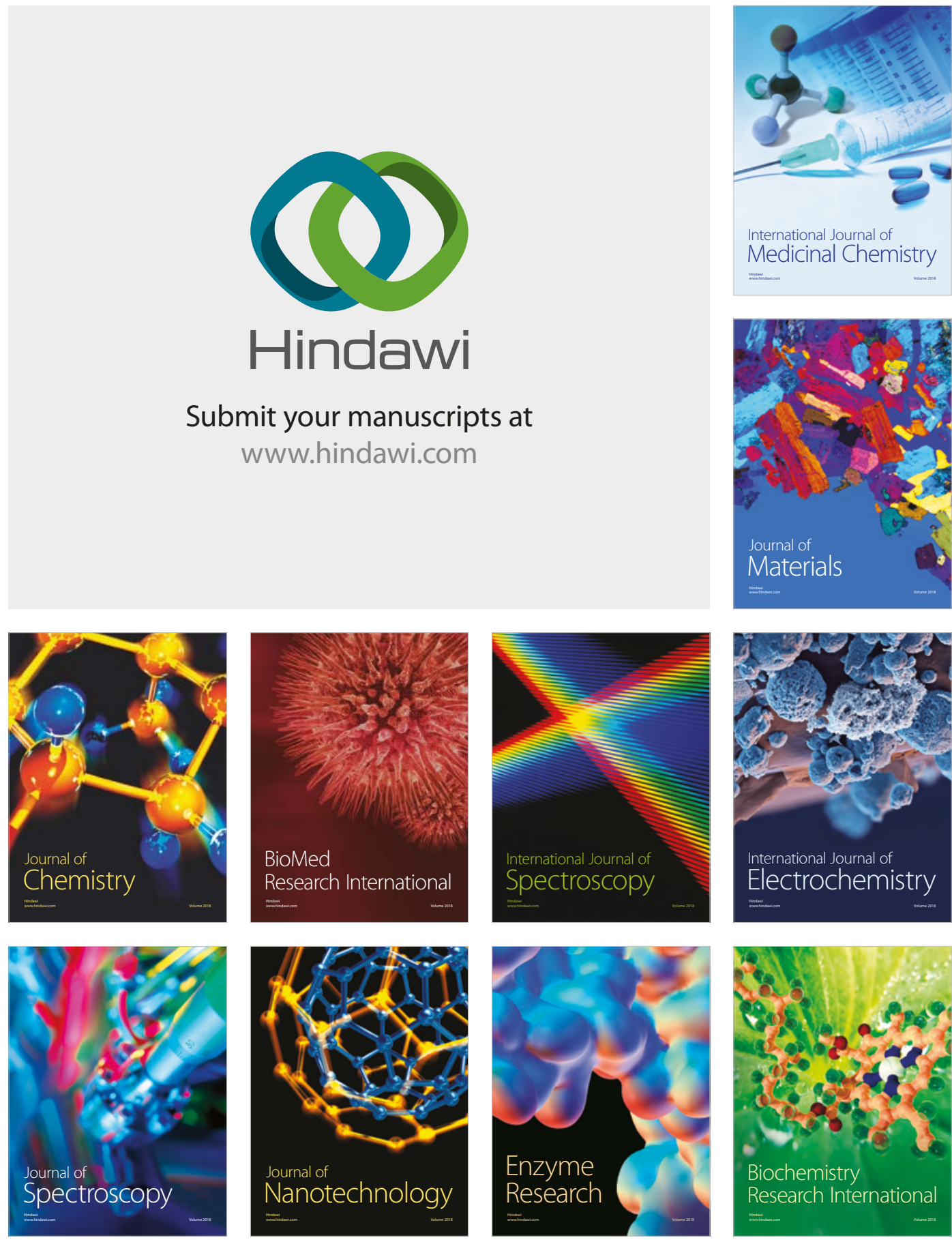
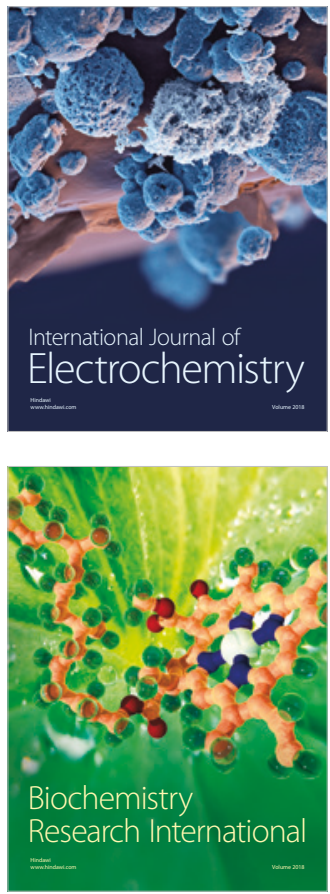